\title{
Gender Differences in Cerebral Cortical Folding: Multivariate Complexity-Shape Analysis with Insights into Handling Brain-Volume Differences
}

\author{
Suyash P. Awate ${ }^{1}$, Paul Yushkevich ${ }^{1}$, Daniel Licht ${ }^{2, \star}$, and James C. Gee ${ }^{1}$ \\ ${ }^{1}$ Penn Image Computing and Science Lab (PICSL), University of Pennsylvania \\ awate@mail.med.upenn.edu \\ ${ }^{2}$ Children's Hospital of Philadelphia, USA
}

\begin{abstract}
This paper presents a study of gender differences in adult human cerebral cortical folding patterns. The study employs a new multivariate statistical descriptor for analyzing folding patterns in a region of interest (ROI) and a rigorous nonparametric permutation-based scheme for hypothesis testing. Unlike typical ROI-based methods that summarize folding complexity or shape by single/few numbers, the proposed descriptor systematically constructs a unified description of complexity and shape in a high-dimensional space (thousands of numbers/dimensions). Furthermore, this paper presents new mathematical insights into the relationship of intra-cranial volume (ICV) with cortical complexity and shows that conventional complexity descriptors implicitly handle ICV differences in different ways, thereby lending different meanings to "complexity". This paper describes two systematic methods for handling ICV changes in folding studies using the proposed descriptor. The clinical study in this paper exploits these theoretical insights to demonstrate that (i) the answer to which gender has higher/lower "complexity" depends on how a folding measure handles ICV differences and (ii) cortical folds in males and females differ significantly in shape as well.
\end{abstract}

\section{Introduction}

Cerebral cortical folding forms an underpinning for the cognitive skills and behavioral traits in humans. For the last few decades, magnetic resonance (MR) imaging has enabled in vivo studies of human cortical folding patterns. One class of approaches to folding analysis rely on spatial normalization 1,2 and subsequently perform statistical hypothesis testing at every voxel or surface element in the normalized space. However, the difficulty in finding sufficiently-many homologous features 34 may directly affect the normalization and, thereby, the

\footnotetext{
* The authors gratefully acknowledge the support of this work via NIH grants HD042974, HD046159, NS045839, EB06266, DA14129, DA22807, UL1RR024234, K23 NS052380, NS061111, K25 AG027785, the Dana Foundation, the June and Steve Wolfson Family Foundation, and the Institute for Translational Medicine and Therapeutics' (ITMAT) Transdisciplinary Awards Program in Translational Medicine and Therapeutics at the University of Pennsylvania.
} 
reliability of findings in the clinical study. Furthermore, the phenomenon of cortical folding has an inherent large-scale or non-local character. A second class of approaches propose region-based folding descriptors 567 , which avoid the challenges associated with normalization by reducing spatial sensitivity from a voxel to a region of interest (ROI) that can be reliably defined in each individual based on observed homologous features.

Most studies in the literature measure only the complexity of folding, ignoring information related to shape, orientation, etc. Although some recent ROI-based approaches propose descriptors incorporating shape information 8 , they fail to integrate all the information on shape and complexity in a single descriptor. Furthermore, typical ROI-based approaches produce scalar or low-dimensional summary statistics for the entire ROI, risking serious information loss. This paper builds on a new ROI-based statistical framework for folding analysis 9] relying on a rich multivariate non-local descriptor that captures the spectrum of complexity and shape. The proposed descriptor is a joint probability density function (PDF) of two variables, one capturing surface complexity and the other capturing surface shape. The paper proposes a new application of a nonparametric permutation-based approach for statistical hypothesis testing with multivariate cortical descriptors. In these ways, the proposed framework couples the reliability of ROI-based analysis with the richness of the proposed descriptor.

While several folding studies concern neurodevelopmental disorders, studies on gender differences, in the normal population, have received very little attention. Moreover, while one study [10] using the fractal-dimension (FD) measure reported higher complexity in adult females, two very recent studies [1112] using the isoperimetric ratio (IPR) measure report higher complexity in larger adult brains (i.e. males). The study in this paper elucidates these seemingly-conflicting findings. This paper provides new theoretical insights into relationships between folding measures with intra-cranial volume (ICV), pinning them down to the fundamental issues of scale and replication. It shows that standard folding measures in the literature imbibe different meanings of "complexity". It shows that handling ICV differences in folding studies may not be as simple as including ICV as a covariate in the underlying statistical test. This paper proposes two systematic methods for handling ICV changes in folding studies using the proposed descriptor and shows that while the findings using one method are consistent with [10], those using the other method are consistent with [1112.

While it is well known [5] that the shape of cortical folds is asymmetric, i.e. surface area buried in sulci (predominantly concave areas) being more than that for gyri, the literature on gender differences in folding ignores studies of shape. This paper is, perhaps, the first to demonstrate that the fraction of the cortical surface that is convex (predominantly gyri) is significantly higher in males.

\section{Background}

This section describes a variety of existing ROI-based folding descriptors summarizing a small part of the complexity-shape spectrum via one or a few numbers. 
One class of descriptors quantify surface complexity alone. FD [10] captures the rate of increase in surface area over multiscale representations of the surface. Gyrification index (GI) 13 is the ratio of the lengths of a planar curve to its envelope. Convexity ratio (CR) [6] is the ratio of the area of the surface to the area of the convex hull/envelope of the surface. IPR [6 11,12] is the ratio of the surface area to the two-third power of the volume enclosed by the surface. Average curvedness (AC) [8] measures the deviation of the surface from a plane. Another measure is the 2D centroid of the histogram (HC) of curvature [7].

Some folding descriptors capture a part of the complexity-shape spectrum by summing up specific measures for all surface patches, e.g. intrinsic curvature index (ICI) 5] sums up degrees of hemisphericity, mean curvature norm $(\mathrm{MCN})$ [6] sums up degrees of hemisphericity and cylindricity, Gaussian curvature norm (GCN) 6] sums up degrees of hemisphericity and saddle-likeness, average shape index (AS) [8] sums up shape measures, etc.

\section{Methods and Materials}

\subsection{A Multivariate High-Dimensional Folding Descriptor}

This section describes a novel high-dimensional multivariate surface descriptor that captures the spectrum of complexity and shape [9].

At every point $m$ on surface $\mathcal{M}$, the principal curvatures $K_{\min }(m)$ and $K_{\max }(m)$ completely describe the local patch geometry. The space $<K_{\min }, K_{\max }>$ can be reparameterized into the orthogonal basis of curvedness $C$ and shape index $S$ [14], meaningfully separating notions of bending and shape (Figure1).

We propose the following generative model of cortical surfaces. Let us consider $C: \mathcal{M} \rightarrow[0, \infty]$ and $S: \mathcal{M} \rightarrow[-1,1]$ as random fields. Let us also consider the joint PDF that captures the dependencies between $C(m)$ and $S(m)$ for a specific class of surfaces. Consider a finite collection $\mathcal{O}=\left\{d \mathcal{M}^{1}, \ldots, d \mathcal{M}^{T}\right\}$ of $T$ surface

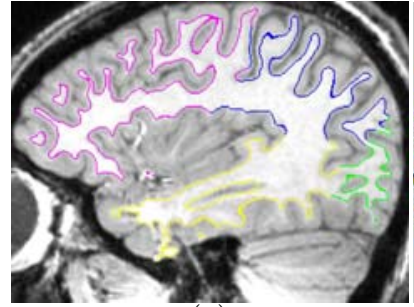

(a)

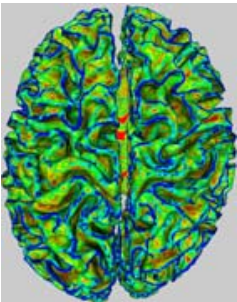

(b)

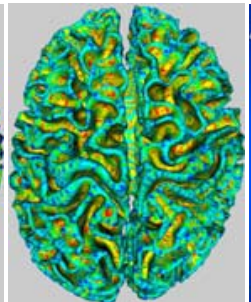

(c)

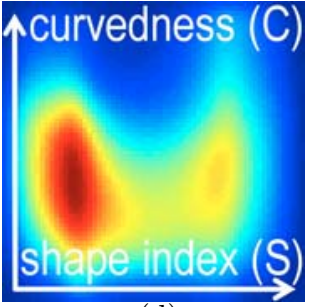

(d)

Fig. 1. (a) A sagittal slice of an MR image overlapped with the zero crossing of the level set that represents the cortical surface. (b) Curvedness $C(m)$ values painted on $\mathcal{M}($ red $\rightarrow$ blue $\equiv$ low $\rightarrow$ high $)$. (c) Shape-index $S(m) \in[-1,1]$ values painted on $\mathcal{M}$ $\left(\right.$ red $\rightarrow$ blue $\equiv-1 \rightarrow 1$ ). (d) Proposed descriptor $P_{\mathcal{M}}(C, S)$ (blue $\rightarrow$ red $\equiv$ low $\rightarrow$ high; for all plots in this paper, horizontal axis $\equiv S$ and vertical axis $\equiv C$ ). 
patches, located at points $\left\{m^{1}, \ldots, m^{T}\right\} \in \mathcal{M}$ uniformly distributed over the surface $\mathcal{M}$, covering $\mathcal{M}$. Then, $\left\{\left(C\left(m^{1}\right), S\left(m^{1}\right)\right), \ldots,\left(C\left(m^{T}\right), S\left(m^{T}\right)\right)\right\}$ is an instantiation of the field of random vectors at locations $\left\{m^{1}, \ldots, m^{T}\right\}$. We assume that the random field is stationary, i.e. each observation $\left(C\left(\mathrm{~m}^{t}\right), S\left(\mathrm{~m}^{t}\right)\right)$, is randomly drawn from a single PDF $P_{\mathcal{M}}(C, S)$. The complexity and variability in cortical folding suggests that dependencies between random vectors $\left(C\left(m^{t}\right), S\left(m^{t}\right)\right)$ and $\left(C\left(m^{s}\right), S\left(m^{s}\right)\right)$ decrease at a fast rate with increasing geodesic distance between locations $m^{t}$ and $m^{s}$. Thus, we assume that the random field is mixing.

We propose the joint $\mathrm{PDF} P_{\mathcal{M}}(C, S)$ as the multivariate high-dimensional descriptor of cerebral cortical folding patterns for surface $\mathcal{M}$ (Figure 1(d)). $P_{\mathcal{M}}(C, S)$ subsumes scalar descriptors like ICI, MCN, GCN, AC, AS, HC. Discretizing $P_{\mathcal{M}}(C, S)$ on an $M \mathrm{x} N$ grid leads to an $M N$-dimensional descriptor. In this paper $M=N=64$.

For a given surface $\mathcal{M}$, we propose to estimate the folding pattern descriptor $P_{\mathcal{M}}(C, S)$ from the sample $\left\{\left(C\left(m^{t}\right), S\left(m^{t}\right)\right): t=1, \ldots, T\right\}$ drawn from a stationary mixing random field. A consistent nonparametric estimate $[15$ for the folding descriptor is the Gaussian mixture: $P_{\mathcal{M}}(C, S) \approx \frac{1}{T} \sum_{t=1}^{T} G_{t}\left(\left(C\left(m^{t}\right), S\left(m^{t}\right)\right), \Sigma_{t}\right)$, where $G\left(\left(\mu_{1}, \mu_{2}\right), \Sigma\right)$ is a $2 \mathrm{D}$ Gaussian kernel with mean $\left(\mu_{1}, \mu_{2}\right)$ and covariance $\Sigma$. Consistency requires an optimal choice of $\Sigma_{t}$, dependent on the $T$, and we employ a penalized maximum likelihood scheme [16] to estimate $\Sigma_{t}$; the literature provides many schemes. Figure1(d) shows a typical $P_{\mathcal{M}}(C, S)$ that is multimodal and unlike standard parametric PDFs, thus justifying nonparametric PDF estimation for reliability. In practice, typical ROIs yield sample sizes $T$ in the range of thousands or tens of thousands, producing robust estimations.

\subsection{A Testing Scheme for Multivariate Histogram Analysis}

This section proposes a new application of a known nonparametric permutationbased approach, i.e. statistical nonparametric mapping (SnPM) [17, for statistical hypothesis testing with $N$ multivariate cortical descriptors in a clinical study, i.e. $\left\{P_{\mathcal{M}^{n}}(C, S): n=1, \ldots, N\right\}$, Unlike typical usage of SnPM for functions on the image or surface domain, which necessitates spatial normalization, we propose to apply SnPM to discretized versions of the cortical descriptors $P_{\mathcal{M}^{n}}(C, S)$. Unlike conventional multivariate histogram analysis (e.g. Hotelling $\mathrm{T}^{2}$ ), SnPM provides the locations (pixels and clusters), in the histogram domain $\langle C, S\rangle$, for significant differences/effects.

\subsection{Complexity and Volume Relationships: New Insights}

This section presents new theoretical insights into (i) relationships between folding and ICV and (ii) different meanings of "complexity" underlying descriptors.

In Figure 2, S1 and S2 occupy equal volumes but S2 has finer-scale features than S1. Desirably so, all measures inform that S2 is more complex than S1.

Now consider surfaces occupying different volumes. ICV increase can be associated with two kinds of effects on cortical surfaces: (i) folds are scaled up/ 


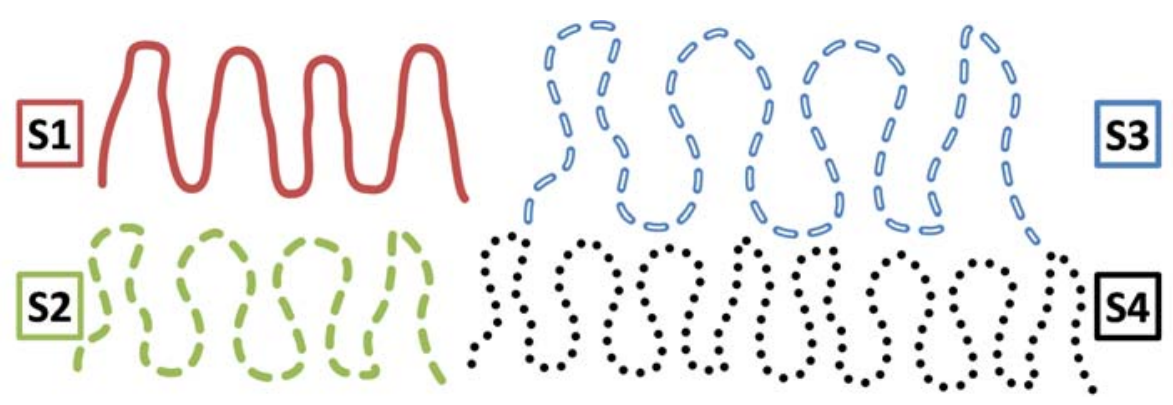

Fig. 2. What does "complexity" mean when volumes differ: issues of scale and replication. S1 and S2 occupy the same volume; S2 is more complex than S1. S3 and S4 occupy larger volumes than S2. S3 enlarges/scales the folds in S2. S4 replicates the folds in S2. How do we compare the complexities of (i) S3 and S2 and (ii) S4 and S2 ?

enlarged, e.g. comparing S2 and S3, or (ii) folds are replicated, e.g. comparing S2 and S4. This section shows that the meaning of "complexity" imbibed in folding descriptors reflects how the descriptors handle scaling and replication.

One class of measures, including GI and CR (both normalized by their convexhull surface area), are invariant to the aforementioned issues of scale and replication. Thus, GI and CR inform that S2, S3, and S4 have equal complexity.

A second class of measures, including IPR, ICI, MCN, GCN, and AC 611128 (all normalized by surface-patch area or, equivalently, $\mathrm{ICV}^{2 / 3}$ ), is designed to be invariant to scale. However, this sacrifices invariance to replication. Thus, these measures inform that S3 and S2 have equal complexity, but S4 is more complex than S2.

A third class of measures, including FD [10, HC [7], and the proposed $P_{\mathcal{M}}(C, S)$ in Section 3.1 are invariant to replication, but not scale. Unlike the first two classes, these measures are not normalized via area or ICV ${ }^{2 / 3}$. Thus, they inform that S4 and S2 are equally complex, but S3 is less complex than S2.

We now propose a new scale-invariant descriptor. Enlarging volume by a factor $\beta^{3}$ reduces curvedness by a factor of $\beta$. Indeed, unit-area patches in enlarged surfaces appear more planar (Taylor's theorem). Thus, a scale-invariant version of $P_{\mathcal{M}}(C, S)$ is $P_{\mathcal{M}}(C \beta, S)$, where $\beta^{3}$ is the ratio of the mean group ICV to the ICV for cortical surface $\mathcal{M}$. Similar to the second class of measures, $P_{\mathcal{M}}(C \beta, S)$ informs that S3 and S2 have equal complexity, but S4 is more complex than S2.

Subsequent sections denote $P^{\text {replication }}=P_{\mathcal{M}}(C, S)$ and $P^{\text {scale }}=P_{\mathcal{M}}(C \beta, S)$.

\subsection{Clinical Cohort, Imaging, and Image Analysis}

The cohort comprised T1 MR images ( $1 \mathrm{~mm}^{3}$ isotropic voxels) of 30 females (age $34.8 \pm 9.6$ years) and 27 males (age $36 \pm 11$ years), obtained after enforcing quality-assurance checks on every image in the dataset in [18].

Image analysis: (i) parcellate lobes, (ii) segment tissues [19], (iii) resample segmentations to $0.4^{3} \mathrm{~mm}^{3}$ isotropic voxels, (iv) fit a level set to the cortical graywhite interface $\mathcal{M}$, (v) compute $(C(m), S(m)) \forall m \in \mathcal{M}$, (vi) estimate $P^{\text {replication }}$ 
(Section 3.1) and $P^{\text {scale }}$ (Section 3.3) for all lobes, (vii) cross-sectional test for gender differences via SnPM (Section 3.2). Preplication has been validated in 9 .

\section{Results and Discussion}

\subsection{Gender Differences in Cortical Complexity and Shape in Adults}

The proposed replication-invariant folding descriptor $P^{\text {replication }}$, described in Section 3.1, produces t maps (Figure 3(a)-(d)) indicating larger histogram mass for males (red) in low-curvedness regions (bottom half) and larger histogram mass for males (red) in convex regions (right half). SnPM produces significant clusters for the occipital lobes (Figure 3(e)), but not for other lobes. Nevertheless, when the ROI size is increased to a hemisphere, these effects get significantly strengthened; evident in t maps (Figure [3(f),(h)) and significant clusters (Figure 3(g),(i)). These results show that female cortical surfaces are more complex based on Preplication (consistent with [10]) and significantly less convex than those of males. Figures $3(\mathrm{j})-(\mathrm{k})$ visualize the complexity differences.

The proposed scale-invariant folding descriptor $P^{\text {scale }}$, described in Section 3.3. produces $t$ maps (Figure 4(c)) indicating larger histogram mass for males (red) in high-curvedness regions (top half) for all lobes. Complexity differences are very strong, producing significant locations (Figure 4(d)) and clusters for all lobes, and overwhelm shape differences. These results show that,

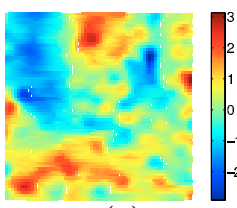

(a)

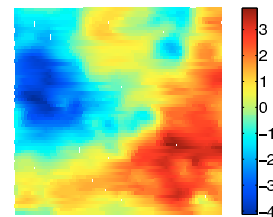

(f)

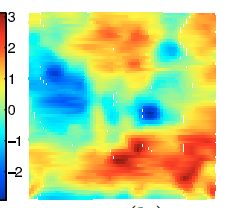

(b)

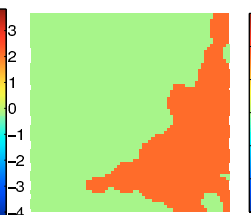

(g)

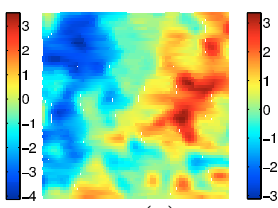

(c)

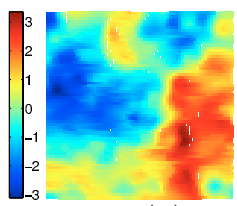

(d)

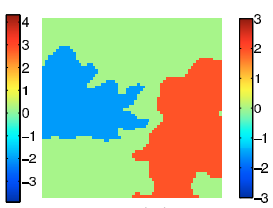

(e)

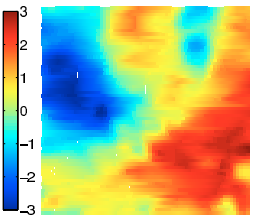

(h)
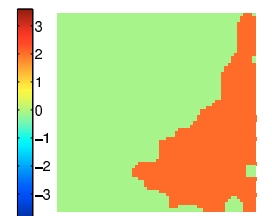

(i)

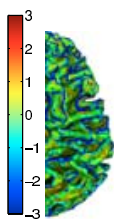

(j)

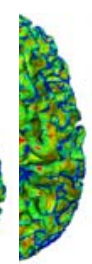

$(\mathrm{k})$

Fig. 3. (a)-(d) Student's t statistics for a cross-sectional study with $P^{\text {replication }}$ (positive $\mathrm{t} \equiv$ larger value in males) for frontal, parietal, temporal, and occipital lobes, respectively, in the left hemisphere. Similar patterns exist for lobes in right hemisphere. (e) Significant clusters via SnPM for left occipital lobe. For all plots in this paper, corrected $p$ values for significant locations/clusters are indicated by coloring them by the associated $z$ score: e.g. $z(p=0.05)=1.65, z(p=0.005)=2.58$. (f)-(h) $\mathrm{t}$ statistics and (g)-(i) significant clusters for $P^{\text {replication }}$ for left hemisphere (4 lobes) and whole brain (8 lobes), respectively. A similar pattern exists for the right hemisphere. (j)-(k) female and male brains, respectively, painted by $C$ values (red $\rightarrow$ blue $\equiv$ low $\rightarrow$ high). The female brain appears more blue/cyan (more "complex"). 


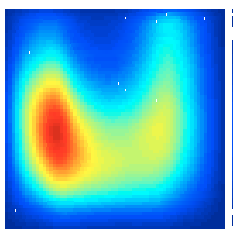

(a)

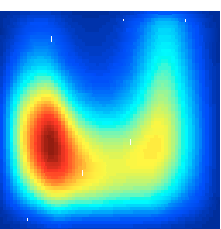

(b)

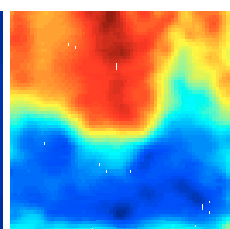

(c)

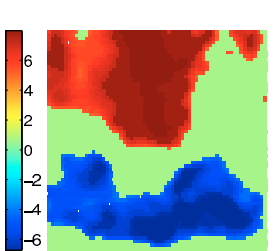

(d)

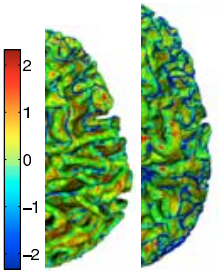

(e) (f)

Fig. 4. (a)-(b) Average of $P^{\text {scale }}$ for males and females, respectively, for the left frontal lobe. (c)-(d) t statistics (positive $\mathrm{t} \equiv$ larger value in males) and significant locations, respectively, for the left frontal lobe. Similar patterns exist for all other lobes. (e)(f) female and male brains, respectively, adjusted for ICV and painted by $C$ values $($ red $\rightarrow$ blue $\equiv$ low $\rightarrow$ high). The female brain appears more red/yellow (less "complex").

when ICV differences have been accounted for (via $P^{\text {scale }}$ ), male cortical surfaces are more complex than those of females. This interpretation is consistent with [112]. Figures 4(e)-(f) help visualize the complexity differences.

This paper exploited two new multivariate high-dimensional (65536 dimensional) folding descriptors, unifying complexity and shape information, to provide new mathematical insights into the different meanings of complexity in the context of ICV differences. The paper exploits these insights to resolve two seemingly-contradictory findings in the state of the art on gender-based cortical folding, i.e. 10 and 1112 differ in which gender has higher "complexity". The cross-sectional clinical study in this paper demonstrates that while the female cortex has more fine-scale features (which is the meaning of "complexity" in [10]), the male cortex has a disproportionately greater bending in proportion to its larger volume (which is the meaning of "complexity" in [11]2]). Thus, the results show that folding patterns in males differ from those in females in two fundamental ways: (i) enlargement/scaling of folds and (ii) additional folds or bending. Amazingly, the magnitude of the latter effect is (i) weak enough to keep the bending in female folds, without any adjustment for ICV, more than the bending in males, but (ii) strong enough to reject the hypothesis that folding patterns in the two genders are simply scaled versions of each other. Furthermore, this paper is perhaps the first to show significant gender differences in gyral/sulcal shape.

Recent studies 20] have found that the female cortex is thicker in some regions even without compensating for lower ICV. After accounting for ICV differences, the entire cortex is significantly thicker in females. The complementary findings concerning cortical complexity, shape, and thickness might help explain the similarities and differences in cognitive skills possessed by both genders.

\section{References}

1. Yu, P., Grant, P.E., Qi, Y., Han, X., Segonne, F., Pienaar, R., Busa, E., Pacheco, J., Makris, N., Buckner, R.L., Golland, P., Fischl, B.: Cortical surface shape analysis based on spherical wavelets. IEEE Trans. Med. Imaging 26(4), 582-597 (2007) 
2. Nordahl, C., Dierker, D., Mostafavi, I., Schumann, C., Rivera, S., Amaral, D., Van-Essen, D.: Cortical folding abnormalities in autism revealed by surface-based morphometry. Journal of Neuroscience 27(43), 11725-11735 (2007)

3. Mangin, J., Riviere, D., Cachia, A., Duchesnay, E., Cointepas, Y., PapadopoulosOrfanos, D., Scifo, P., Ochiai, T., Brunelle, F., Regis, J.: A framework to study the cortical folding patterns. NeuroImage 23(1), S129-S138 (2004)

4. Van-Essen, D., Dierker, D.: Surface-based and probabilistic atlases of primate cerebral cortex. Neuron. 56, 209-225 (2007)

5. Van-Essen, D., Drury, H.: Structural and functional analyses of human cerebral cortex using a surface-based atlas. J. Neuroscience 17(18), 7079-7102 (1997)

6. Batchelor, P., Castellano-Smith, A., Hill, D., Hawkes, D., Cox, T., Dean, A.: Measures of folding applied to the development of the human fetal brain. IEEE Trans. Med. Imaging 21(8), 953-965 (2002)

7. Pienaar, R., Fischl, B., Caviness, V., Makris, N., Grant, P.E.: A methodology for analyzing curvature in the developing brain from preterm to adult. Int. J. Imaging Systems Technology 18(1), 42-68 (2008)

8. Awate, S.P., Win, L., Yushkevich, P., Schultz, R.T., Gee, J.C.: 3D cerebral cortical morphometry in autism: Increased folding in children and adolescents in frontal, parietal, and temporal lobes. In: Proc. Int. Conf. Medical Image Computing and Computer Assisted Intervention, vol. 1, pp. 559-567 (2008)

9. Awate, S.P., Yushkevich, P., Song, Z., Licht, D., Gee, J.C.: Multivariate highdimensional cortical folding analysis, combining complexity and shape, in neonates with congenital heart disease. In: Proc. Information Processing in Medical Imaging (to appear, 2009)

10. Luders, E., Narr, K., Thompson, P., Rex, D., Jancke, L., Steinmetz, H., Toga, A.: Gender differences in cortical complexity. Nat. Neuro. 7(8), 799-800 (2004)

11. Im, K., Lee, J., Lytteleton, O., Kim, S., Evans, A., Kim, S.: Brain size and cortical structure in the adult human brain. Cer. Cor. 18, 2181-2191 (2008)

12. Toro, R., Perron, M., Pike, B., Richer, L., Veillette, S., Pausova, Z., Paus, T.: Brain size and folding of the human cerebral cortex. Cer. Cor. 18, 2352-2357 (2008)

13. Zilles, K., Armstrong, E., Schleicher, A., Kretschmann, H.: The human pattern of gyrification in the cerebral cortex. Anat. Embryol. 179, 173-179 (1988)

14. Koenderink, J., van Doorn, A.: Surface shape and curvature scales. Image and Vision Computing 10(8), 557-565 (1992)

15. Lu, Z., Chen, X.: Spatial kernel regression estimation: weak consistency. Stat. and Prob. Letters 68(2), 125-136 (2004)

16. Chow, Y., Geman, S., Wu, L.: Consistant cross-validated density estimation. Annals of Statistics 11(1), 25-38 (1983)

17. Nichols, T., Holmes, A.: Nonparametric permutation tests for functional neuroimaging: a primer with examples. Human Brain Mapping 15(1), 1-25 (2002)

18. Mortamet, B., Zeng, D., Gerig, G., Prastawa, M., Bullitt, E.: Effects of healthy aging measured by intracranial compartment volumes using a designed MR brain database. In: Med. Imag. Comput. Comp. Assist. Interv, pp. 383-391 (2005)

19. Awate, S.P., Tasdizen, T., Foster, N.L., Whitaker, R.T.: Adaptive Markov modeling for mutual-information-based unsupervised MRI brain-tissue classification. Medical Image Analysis 10(5), 726-739 (2006)

20. Sowell, E., Peterson, B., Kan, E., Woods, R., Yoshii, J., Bansal, R., Xu, D., Zhu, H., Thompson, P., Toga, A.: Sex differences in cortical thickness mapped in 176 healthy individuals between 7 and 87 years of age. Cer. Cor. 17, 1550-1560 (2007) 